\title{
Musique et harmonie des quatre mondes ou le cercle IV de la Galliade de G. Lefêvre de la Boderie
}

\author{
S. MASER
}

La Renaissance a vu une floraison de traités musicaux, certains écrtis par des musiciens réfléchissant sur leur art mais bien plus par des humanistes qui pensaient qu'il était possible de restaurer la musique antique. Le premier de ces humanistes est Gafori ${ }^{1}$ avec sa Theorica musicae publiée en 1492. La liste des théoriciens italiens est déjà longue lorsqu'en France, Tyard fait apparaître le Solitaire second ou Prose de la Musique en 1552. C'est un quart de siècle plus tard que Guy Lefèvre de la Boderie parlera à son tour de musique dans le Cercle IV de sa Galliade (1578). Il faudrait, pour compléter le tableau des "musicologues" français de la Renaissance ajouter deux noms: ceux de Mersenne (Quaestiones Celeberrimae in Genesim 1623 et surtout L'Harmonie universelle 1636) et Bergier (La Musique spéculative) ${ }^{2}$ Bien que ces deux derniers auteurs appartiennent au XVII ${ }^{\mathrm{e}}$ siècle, leurs conceptions musicales sont de la Renaissance.

Avant de procéder à un examen de la théorie musicale de Guy Lefèvre de la Boderie, arrêtons-nous un instant pour essayer de comprendre pourquoi des humanistes aussi différents qu'un Tyard, un Mersenne, un Zarlino, un Glarean, un Guy Lefèvre de la Boderie - pour ne citer que quelques noms se sont intéressés à la musique.

La musique, tout d'abord, n'est pas considérée comme un art, similaire à la peinture par exemple; elle fait partie intégrante du quadrivium, elle est une "des quatre voies" à côté de l'arithmétique, le géométrie et l'astrologie qui mènent à la philosophie selon Platon ou qui constituent une branche même de la philosophie selon la tradition aristotélienne. Les humanistes, découvrant, redécouvrant les textes antiques sont heureux de trouver chez Platon, Plutarque ou Ptolémée ce qu'ils avaient appris chez Boèce (De Institutione Musica), Cassiodorus (De Artibus ac Disciplines Liberalium Litterarum), Saint Augustin (De Musica), Aristote et Macrobe (Commentarium in Somnium Scipionis). Comme chez les penseurs médiévaux on se délecte à établir de mystérieuses correspondances entre la musique des sphères (musica mundana), la musique de l'homme (musica humana) et la musique créée par des instruments (musica instrumentis constituta) (une preuve de plus, s'il était nécessaire, de la non rupture entre la pensée médiévale et la 
pensée humaniste!). S'il y a correspondances entre le macrocosme et le microcosme - le Petit Monde comme l'appelle Guy Lefèvre de la Boderie on doit pouvoir, par l'étude, instaurer chez l'homme une harmonie parallèle à celle que l'on peut admirer dans l'univers. Grâce à cette harmonie, cet équilibre, la paix universelle pourra enfin régner. Tous les humanistes se sont penchés sur les "effets" produits par la musique, espérant amener cette paix qui faisait tant défaut dans ces pays ravagés par les guerres civiles et religieuses. Cette aspiration, cette foi dans les effets de la musique expliquent entre autre l'entreprise malheureuse de J.A. de Baïf d'écrire de la poésie en vers mesurés ainsi que la fondation de son Académie de poésie et musique.

Un traité sur la musique trouvait naturellement sa place dans une somme encyclopédique comme La Galliade écrite à l'honneur de la Gaule. Après avoir "remarié le Ciel avec la Terre" c'est-à-dire attribué les soixante-douze pays originels aux douze signes du Zodiac suivant l'exemple de G. Postel, Guy Lefèvre de la Boderie étudie l'architecture puis la magie - science où les druides ont excellé. Enfin, dans un quatrième cercle il traite "de la Musique et Harmonie, tant du Monde archétype, Celeste et élémentaire, que de celle de l'Homme ou du Petit Monde, et des merveilleux effets qu'elle produit."'3 L'intérêt pour la musique de G. Lefèvre de la Boderie est, on le voit, semblable à celui de tous les autres humanistes. Il s'agit d'harmonie, la ligne mélodique pas plus que le rythme n'attire son attention. Il s'agit d'établir quels accords sont harmonieux, quels intervalles rendent les sons consonnants ou dissonnants. Guy Lefèvre de la Boderie s'efforcera de révéler les relations entre l'Univers et le "petit monde" et la dernière partie de son exposé sera consacré aux effets de la musique. Comme La Galliade est, outre une somme encyclopédique, un hymne à la gloire de la Gaule, on trouvera d'abord un exposé affirmant la supériorité de la Gaule sur toutes les autres nations puisqu'elle est, assure-t-il, le berceau de la musique.

\section{La Gaule, berceau de la musique}

Dans un double élan de retour aux sources et de patriotisme, bon nombre d'écrivains tels Guillaume Postel, Jean Lemaire, Corrozet, Jean Bouchet etc. se font gloire d'être descendants de Gaulois. C'est que, se fondant sur la généalogie fantaisiste de Bérose, ils ne comptent pas moins que Noé dans leurs ancêtres par l'intermédiaire de Japhet. Outre cette illustre origine, les Gaulois ont été fameux - toujours au dire de ces humanistes - pour avoir été les premiers à inventer les sciences et les arts, dont la musique!

A Drius, et aux siens, cinquiesme au rang des Rois Qui tindrent en leur main le sceptre des Gaulois

Son fils Bardus succède, auquel la Poèsie

Et Musique sa soeur, couple heureuse et choisie

Doit son commencement, ainsi que rondement 
Résonne son beau nom le fils du Fondement

Et peut bien s'exposer en bonne convenance

Le vray nom de Bardus, fils de la Résonnance (p. 77 r)

Pour appuyer sa thèse, Guy Lefevre de la-Boderie a recours à l'émythologie. Bardus viendrait de l'araméen bar dusa בר דאושא, bar signifiant fils et $d u$ sa fondement ou bien de bar/dus בר דאוש fils de reconnaissance ou bien de résonnance. Nos bons vieux Poètes, les Bardes, descendants de Bardus ont inventé les orgues, les violes, le luth, la harpe et la lyre. Plus important encore, peut-être, ils ont allié avant les Grecs, la musique à la poésie afin de mieux vanter la vertu et condamner d'une façon irrémédiable le vice. Ainsi les vieux Gaulois connaissaient-ils cette heureuse union entre musiciens, poètes et sages, union qu'a tant recherchée Baïf par exemple en France ou Zarlino de la Carmerata florentine. Grâce à cette union, les Bardes ont été à même d'apaiser les esprits:

l'orgueil, la haine, l'ire

Tomboit au seul regard des peres de la lyre

Et tous les hurlements et les cris odieux

Cedoient à leurs accords doux et mélodieux ... (p. 78 r)

Guy Lefèvre de la Boderie insiste sur l'antériorité des arts chez les Gaulois. En Bourgogne il existe, dit-il, un mont nommé Barré dans lequel on n'aura pas de peine à y lire de mot Barde:

Là les Muses en rond menèrent leurs carolles Avant que Grèce sceust aformer les parolles Et maints siecles avant que l'Assocan berger Eust songé de les voir sur Barnasse Leberger Voire avant qu'eust senty leur mere Moremosyne

En Grèce, les douleurs de la femme en gésine. (p. 78 v)

Et comme La Galliade est un hymne à la gloire de la Gaule, il n'oublie pas de parsemer son poème d'allusions à cette antériorité, comme on le verra au cours de l'exposé. Pythagore, à qui l'on a l'habitude d'attribuer la découverte des lois concernant les intervalles musicaux, se révèle bien gênant; alors, dans bien des cas, il taira son nom, pourtant bien cher pour un néoplatonicien. Enfin, croyant en la vertu de la répétition comme moyen du persuasion, Lefèvre de la Boderie conclut son cercle IV en louant encore une fois les Bardes d'avoir su allier la musique à la poésie:

Ainsi, de main en main la Musique choisie

Qui anime, et qui meut la douce Poésie,

Et la porte dans l'air sur ses accords ailez,

Print son commencement des Bardes oreillez,

Qui les premiers ont joint les vers à la Musique 
Que du los des Herôs ils ont fait héroïque:

Premiers ils ont les vers aux nombres alliez,

Et les nombres et vers aux chordes mariez

Et premiers ont sonné sur les Gauloises rives

Des morts par nom vivants les gloires tousjours vives (p. 107 r)

\section{Musique et Harmonie}

Une fois affirmée la précédence de la Gaule sur les autres pays, l'auteur peut passer à son exposé théorique sur la musique. Guy Lefèvre de la Boderie ne se pose pas en musicien ou en simple amateur de musique. Ce n'est pas un traité d'esthétique qu'il veut nous présenter. La musica practica ne l'intéresse pas davantage qu'elle n'a intéressé les exposants platoniciens et néoplatoniciens de la musique. La musica practica ne concerne que les artisans, capables de produire des sons flatteurs à l'oreille mais incapables de comprendre la raison interne de l'harmonie, en un mot les empiristes. Le philosophe ne saurait se contenter d'étudier le système musical sous le point de vue de l'esthétique mais il étudiera avant tout les rapports des sons entre eux pour déterminer "scientifiquement," raisonnablement quels sons s'accordent et quels sons produisent un accord discordant. Le nombre est la clé du système harmonique platonicien hérité du système pythagoricien. Il est évident que c'est le côté spéculatif qui attire l'auteur de La Galliade.

Avant d'aborder l'étude de la consonnance, il doit définir le son. Pour Platon (Le Timée) et Aristote (De Anima) le son était comparable à un projectile qui atteignait l'âme. C'est Boèce que Guy Lefèvre de la Boderie suit. En effet, il met en évidence le mouvement vibratoire du son et l'importance de l'air comme milieu de transmission:

. . . Le son produit de la bouche une fois

Se respand dedans l'air, et y descrit et trace

Tout autour de son poinct meint cercle et meint espace (p. $78 \mathrm{v})$

Il emprunte même à Boèce l'image d'une eau dormante troublée par le jet d'un caillou:

Ne plus ne moins qu'on voit qu'en calme et dormante eau
Le seul ject d'un caillou meint et meint cercle beau,
L'un de l'autre suivy tousjours en forme ronde
Trace, peint et descrit sur le Tableau de l'onde (p. $78 \mathrm{v}$ )

Il fait dépendre la qualité du son du mouvement comme l'avaient fait, avant lui, Boèce et d'autres harmoniciens:

Bas, quand il est poussé tout tardif et tout lent

En haut, quand il se fait soudain et violent (p. 79 r)

et répète la théorie erronée que l'on trouve chez Macrobe. Celui-ci soutient que dans une flûte de pan le son est d'autant plus aigu qu'il est près de sa 
source. Nous touchons là à un aspect important de l'attitude philosophique des humanistes et principalement des néo-platoniciens: ils reçoivent et retransmettent sans aucun esprit critique la "vérité" qu'ils ont découvert dans les écrits révérés des anciens. C'est comme une "révélation" qu'ils s'interdisent d'examiner. Macrobe n'était pas le seul à soutenir cette opinion. On la trouve bien sûr chez Cicéron dont Macrobe commente le Songe de Scipion. Guy Lefèvre de la Boderie passe très rapidement de cette musica humana à la musica mundana qui intéresse au premier chef notre auteur. Il établit un parallèle entre ces deux sortes de musique:

Ne plus ne moins le son qui sort comme du centre

Du grand Esprit moteur, et dans les Sphères entre

Se fait ouyr plus haut aux Esprits despouillez

De la masse du corps, et des sens embrouillez,

D'autant qu'il est plus près de son principe et source,

Et d'autant que le Ciel haste plustost sa course (p. 79 r)

On aura reconnu au passage le dogme platonicien de l'âme du monde communiquant l'impulsion qui met en marche l'univers. Le ciel le plus haut, le dixième, habité par Apollon est celui qui résonne le plus clair et le plus haut; et en passant graduellement par les neuf Syrènes ou Muses, on arrive:

Jusqu'au ciel de la lune, où la voix estendue

est plus lente et plus foible, et moins clair entendue. (p. 79 r)

Se rangeant dans la lignée des Platoniciens qu'il cite en marge de son poème ("voy le Timee de Platon, dit-il, et les Commentaires de Proclus et Ficin, et Macrobe sur le Songe de Scipion"), Lefèvre de la Boderie explique comment l'âme de l'Univers se compose des sept nombres, divins mâles et femelles:

Car l'Ame, disoit-il, qui comble l'Univers

Se lie et s'entretient de sept nombres divers

De nompair et de pair comme masle et femelle

Ensemble mariez d'alliance gemelle. (p. 79 v)

Ce passage nous remet en mémoire l'exposé de Cicéron dans le Songe de Scipion: "Hinc Plato postquam et Pythagoricae successione doctrinae et ingenii proprii divina profundidate cognovit nullam esse passe sine his numeris jugabilem competentiam, in Timaeo suo mundo animan per istorum numerorum contextionem ineffabili providentia dei fabricatoris instituit" (Somnium 2, 2.1) où nous voyons l'âme du monde tissée de ces nombres découverts par Pythagore. Dans le poème de Lefèvre de la Boderie si permué de culture antique nous remarquons l'absence éloquente de Pythagore. Alors que tous les théoriciens de la musique, Boèce, Cicéron, 
Macrobe, Nicomaque, Proclus, etc. se délectent à relater la découverte de l'harmonie par Pythagore, l'auteur de La Galliade ne mentionne pas ce nom prestigieux. C'est qu'attribuer la découverte de l'harmonie à Pythagore irait à l'encontre du gomérisme qu'il professe; non, l'origine de la musique doit se voir chez Bardus, en Gaule, et non en Grèce.

Les sept nombres qui ainsi tissent l'âme universelle forment une pyramide ayant au sommet "La saincte unité." Cette pyramide représente la combinaison de deux progressions géométriques, l'une de raison 2 (binaire) et l'autre de raison 3 (ternaire). Déjà avant Platon, Crantor avait figuré cette combinaison sous la forme d'un lambda.

Donq le nombre premier qui s'assied et se met

Ainsi qu'en Pyramide à la poincte et sommet

Est la saincté Unité: après suit le Binaire

Arrengé d'une part, de l'autre le Ternaire, etc. (p. 79 v)

Il suit l'ordre indiqué par Platon dans Le Timée, à savoir 1-2-3-4-9-827 ce qu'on pourrait représenter ainsi:

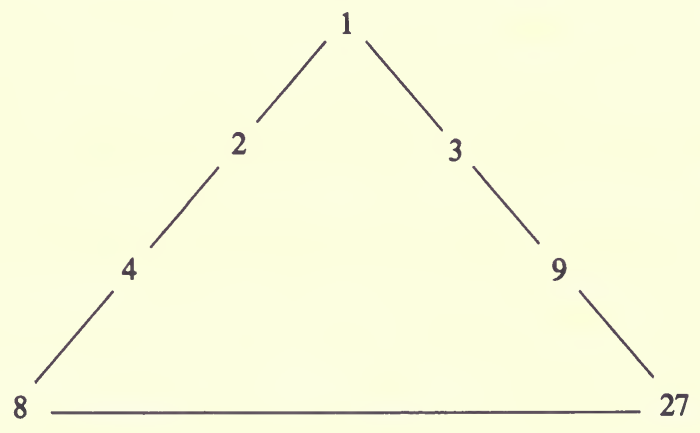

8 - 27 forment la base du triangle ce que dit explicitement notre auteur:

Puis huit succède après, qu'est double au quatrième

Et octuple au premier, et vis à vis de luy

Pour fonder le Triangle en sa base et appuy

Est assis vingt et sept, triple à neuf, et qui plie

L'Un, que vingt et sept fois dedans soy multiplie. (p. $79 \mathrm{v}$ )

Nous voyons ainsi formée "la grande tetraktys" où la somme des six premiers nombres est égale au septième $(1+2+3+4+9+8=27)$ et qui est si chère aux Pythagoriciens. Nous avons aussi remarqué le caractère exceptionnel de l'unité, puisqu'elle est "saincte"; or nour savons la place spéciale qu'accordaient les pythagoriciens au nombre un. Celui-ci, étaient pour eux, le principe de tous les nombres pairs ou impairs. Ces sept "beaux nombres" se combinent pour donner six genres d'accord établis sur six rapports que nous présentons dans le tableau suivant: 


\begin{tabular}{|c|c|c|c|}
\hline Rapports & Nombre & Intervalle en grec & Intervalle en français \\
\hline $2 / 1$ & Doublée & Diapason & Octave \\
\hline $3 / 2$ & Hemiole & Diapenté & Quinte \\
\hline $4 / 3$ & $\begin{array}{l}\text { Epitrite } \\
\text { (terme grec pour } \\
\text { sesquitertien) }\end{array}$ & Diatessaron & Quarte \\
\hline $3 / 1$ & & $\begin{array}{l}\text { Diapason et } \\
\text { diapente }\end{array}$ & Octave + quinte \\
\hline $4 / 1$ & & Disdiapason & $\begin{array}{l}\text { Deux fois parfait } \\
\text { Deux octaves }\end{array}$ \\
\hline $8 / 9$ & Epogdoüe & & Un ton \\
\hline
\end{tabular}

Il devrait normalement inverser ce dernier rapport $(9 / 8)$ pour trouver le ton mais il suit la progression linéaire inventée par Théodore, comme l'a également fait Boèce. En effet, le ton est défini comme la différence entre le quinte et la quarte, soit $3 / 2-4 / 3$ (pour trouver la différence, il faut multiplier les termes opposés soient $9 / 8){ }^{4}$

Une fois établi le rapport pour le ton, Lefèvre de la Boderie peut étudier les différents intervalles selon le nombre de tons et demi-tons; mais on notera qu'il ne s'est pas soucié de définir le rapport pour le demi-ton. ${ }^{5}$ Ainsi la quarte est de deux tons et demi, l'octave de six tons entiers, ce qui est vrai si on la décompose ainsi: cinq tons et deux demi-tons. Nous ne nous arrêterons pas sur tous les intervalles qu'il décrit, cela risquerait d'être fastidieux. Toutefois nous nous attarderons, à l'instar de l'auteur de La Galliade, sur la quinzième appelé aussi disdiapason.

l'accord supreme

de la Musique humaine, et le plus estendu

Soit de vent, soit de voix, ou bien du nerf tendu

que l'on puisse imiter et que l'humaine oreille

Puisse en soy percevoir. (p. 80 r et v)

Nous voyons qu'il répète l'erreur courante que l'oreille ne peut percevoir audelà de deux octaves, confondant la perception des sons et la tessiture d'une voix; là encore le manque d'esprit critique est à noter. Comme les autres harmoniciens, il établit une comparaison avec la musica mundana. Ciceron in Somn 2, 1, 24 avait affirmé: "ultro autem se tendit harmoniae caelestis accessio, id est usque ad quater $\delta\llcorner\alpha \pi \alpha \delta \omega \tau$ xaı $\delta ı \alpha \pi v \vartheta \varepsilon "$ Lefèvre, lui, dit:

Mais des Cieux la merveille

S'estend bien plus avant, et peut dessous la voix

Du grand Esprit infus jusques à quatre fois

Comprendre et embrasser par nombreuse ordonnance

Les neuf tons et demy en bonne consonance (p. $80 \mathrm{v}$ ) 
Il semble qu'il ait lu bien rapidement Cicéron qui accordait à l'harmonie céleste une amplitude de quatre octaves plus une quinte, tandis qu'il attribue une amplitude de quatre douzièmes, multipliant par quatre la quinte, elle aussi. Le texte de base pour tous ces commentaires est évidemment $L e$ Timée qui fait reposer la démonstration sur les sept divins nombres et auxquels Lefèvre de la Boderie lui-même fait référence:

Or la suite de voix, ou l'estente de vent

Par marches et degrez ne parvient plus avant

Que jusques au Septième, et Dieu qui tout dispose,

Fait qu'au nombre de Sept presque tout se repose. (p. 80 v)

Tous les nombres $1,2,3,4,9,8,27$ se combinent en effet ainsi, comme le montre Proclus dans son commentaire sur Le Timée:

$1 / 2$ ( $1^{\mathrm{er}}$ octave), $4 / 2$ ( $2^{\mathrm{e}}$ octave), $8 / 4$ ( $3^{\mathrm{e}}$ octave), $16 / 4$ ( $4^{\mathrm{e}}$ octave)

$24 / 16$ (soit $3 / 2$, une quinte) et $27 / 24$ (soit $9 / 8$ un ton).

Les sept nombres sont ainsi impliqués dans la construction de l'harmonie céleste y compris le nombre 27; notre auteur aurait dû alors attribuer à cette harmonia mundi une amplitude de quatre octaves plus une quinte plus un ton.

\section{Musique des Sphères}

Les bases arithmétiques bien établies, Guy Lefèvre de la Boderie peut enfin exposer sa théorie de la musica mundana. Il a certes besoin d'un répondant et en marge de son poème il nous renvoie à Boèce, la plus grande autorité musicale d'alors. Il est intéressant toutefois de remarquer que Boèce passe rapidement sur les correspondances entre les sphères célestes et les sons ${ }^{6}$ tandis que l'auteur de La Galliade s'étale complaisamment sur des pages et des pages. De fait, il a recours davantage à Cicéron, Macrobe et Platon luimême. Il présente deux traditions différentes, toutes les deux cependant suivent l'ordre chaldéen. L'une présente la gamme ascendante dans le sens étoiles-terre:

\begin{tabular}{|c|c|}
\hline \multicolumn{2}{|c|}{ terre } \\
\hline lune & la \\
\hline $\left.\begin{array}{l}\text { mercure } \\
\text { vénus }\end{array}\right\}$ & $\mathrm{sol}^{7}$ \\
\hline soleil & fa \\
\hline mars & $\mathrm{mi}$ \\
\hline ciel d'après & ré \\
\hline turne & It \\
\hline
\end{tabular}

(l'on peut identifier le ciel d'après avec Jupiter) 
C'est la thèse de Nicomaque; tandis que l'autre (représentée par Cicéron) affirme que Saturne entonne le son le plus élevé. L'auteur suggère qu'il y a moyen de concilier ces deux points de vue:

Mais les autres plus murs, qui d'un bon contrepois
Ensemble ont balance la nature des vois
Sourdes et enrouées, hautes claires et nettes,
Avec les qualités propres aux Sept Planètes,
Ont trouvé une voye et un sentier moyen
Pour lier leurs accords d'un plus parfait lien. (p. 81 r)

Lefèvre de la Boderie introduit à ce point une autre explication de l'harmonie céleste et des intervalles; celle-ci est fondée sur la sécheresse et la chaleur des étoiles comme chez Aristide Quintitien. Il attribue de plus des qualités morales aux planètes et démontre quels intervalles sont ou non discordants. Saturne ou Phénom est froid et sec. C'est la planète aux antipodes même de la vie et soumise aux influences maléfiques. On lui attribue donc la seconde et neuvième qui sont des dissonances. Jupiter, appelé Phaeton ou Zedec est à l'opposé, chaud, humide et débonnaire. Pline avait déjà fait remarquer le côté bienfaisant de cette planète (livre I, 6). La voix est donc "grave, agréable et constante" et on lui attribue: "L'octave et la quinzième en parfait accordance" (p. 81 r). Pyrois (Mars) est sec, chaud et malin; seul Vénus, son épouse dans la mythologie, sait l'apaiser. Les anciens lui ont attribué les sons aigus, stridents et les dissonances de la septième et quatorzième. Le soleil vient ensuite chaud et sec mais bénin aux sons "purs, vénérables, et doux, sonores, non-obscurs." On lui attribue la sixième et la treizième qui font partie des consonances, comme le montre Mersenne, ${ }^{8}$ mais qui à cause de leurs rapports $(8 / 5$ et $16 / 15)$ ne sont pas parfaites.

Et entre les accords comme sienne il affecte

La Siziesme et Treiziesme en tous points non parfaite (p. $81 \mathrm{v})$

Vient ensuite Vénus, chaude, humide et douce; elle attire toutes les planètes hors Saturne qui demeure "froid, triste, pesant et morne." Les sons qu'elle produit sont lascifs et amoureux - et on lui attribue la douzième et la quinzième, consonances parfaites. Mercure se fait remarquer par sa diversité:

Avec malins malin, et avec les bons bon.

Pour ce on luy a donné les sons de plusieurs sortes, (p. 81 v)

il jouit d'intervalles à consonances imparfaites tels la quarte et la onzième. $(4 / 3$ et $8 / 3)$. La lune argentée, humide, froide et bonne

Entre grave et agu, qui argentin et plein cause le son qui sonne.

N'est ny par trop tardif, ny aussi trop soudain

Ainçois d'une teneur égale et balancée. (p. 82 r) 
et reçoit les accords de dizième et de tierce, consonants mais non parfaits.

En résumé toutes les planètes sauf deux se voient attribuer des consonances plus ou moins parfaites (Jupiter et Vénus ont des consonances parfaites et le Soleil, Mercure et la Lune des consonances non parfaites) tandis que Saturne, planète maléfique par excellence, reçoit les dissonances de seconde et neuvième et Mars celles de la septième et quatorzième.

Les "harmoniciens" de l'antiquité ne se sont pas bornés à étudier les sons, les accords rendus par les planètes ainsi que leur psychogonie. Ils ont voulu aussi, comme Cicéron, Macrobe, Calcidius, établir les distances des planètes entre elles. Guy Lefèvre de la Boderie donne comme source de référence L'Histoire naturelle de Pline le Vieux et principalement le livre II, chapitres 21-23. Il le suit en effet de très près indiquant la distance de la terre à la lune, soit 126.000 stades et précisant qu'un stade équivaut à 125 pas ou 625 pieds. ${ }^{9}$ Il faut pourtant remarquer deux différences. La première, essentielle, est que Pline évoque plusieurs fois le nom de Pythagore qu'il qualifie de "vir sagacis animi" tandis que l'auteur de La Galliade se garde bien de mentionner ce nom prestigieux comme s'il voulait que ses lecteurs oublient Pythagore afin de mieux révérer Bardus, l'ancêtre des Gaulois! et pourtant au dire de Pline, c'est Pythagore qui a révélé les correspondances entre les tons et les distances des planètes entre elles. La deuxième différence consiste dans le fait que Pline attribue sept tons à la gamme tandis que Guy Lefèvre de la Boderie prend soin de n'attribuer que six tons à la gamme:

Ainsi du ciel huictesme à nos terrestres parcs

De distance il y a six tons entiers espars,

D'ou vient Diapason parfaite symfonie

Qui le grand luth du monde emplit tout d'harmonie. (p. 82 v)

Voici en schéma les distances relatives des planètes entre elles:

\begin{tabular}{ccccccccc} 
Terre & Lune & Mercure & Vénus & Soleil & Mars & Jupiter & Saturne & Zodiaque \\
\hline 1 ton $1 / 2$ & $1 / 2$ & $1-1 / 2$ & 1 & $1 / 2$ & $1 / 2$ & $1 / 2$
\end{tabular}

De la terre au soleil il y a donc une quinte et du soleil au Zodiaque une quarte. Pline avait attribué un ton et demi entre Saturne et le Zodiaque et arrivait aussi au total de sept tons. Lefèvre de la Boderie précise ici qu'il s'agit de la gamme diatonique, la seule qui convienne à l'harmonie du monde et que, seule, la Raison, grâce aux nombres, a pu nous révéler.

La musique est bien une sorte de révélation. Le roi David prenant modèle sur les dix Sephiroth a inventé le parfait décachorde:

Jadis le Chantre et Roy Profète des Hébrieux

Sur les dix Sephiroth, qui sont sur les dix Cieux,

Accorda les accords du parfait décachorde (p. 83 r) 
et, avant lui, Orphée monta son Heptachorde sur le modèle de la lyre de Ciel, tendant sept cords à l'instar des sept errants (planètes); toutefois l'honneur de la Gaule l'exige - avant David, avant Orfée, les vieux Bardes avaient été ravis par la musique céleste.

Mais le céleste son nos vieux Bardes ravit

Et devant mon Orfée, et devant mon David (p. 83 r)

Revenons au Harpeur de Thrace, il a inventé son Heptachorde en s'inspirant de la lyre céleste: à la lune correspond l'hypaté, la corde la plus grosse et la principale; puis moins grosse vient la parhypaté correspondant à Mercure. Sa troisième s'appelle la lichanos (lycanos) et représente Vénus. Au milieu se trouve la mèse qui renvoie au Soleil; proche de la mèse la paramèse associée à Mars; à Jupiter correspond la Paranète et enfin à Saturne, la Nète, la corde donnant le son le plus haut. Pour plus de clarté nous transposons toutes ces données dans le schéma suivant:

$\begin{array}{ll}\text { hypaté } & \text { lune } \\ \text { parhypaté } & \text { mercure } \\ \text { Lichanos } & \text { vénus } \\ \text { Mèse } & \text { soleil } \\ \text { Paramèse } & \text { mars } \\ \text { Paranète } & \text { Jupiter } \\ \text { Nète } & \text { Saturne }\end{array}$

L'on remarquera qu'ici Guy Lefèvre de la Boderie suit l'ordre de Cicéron dans lequel Saturne correspond à la corde la plus haute. Grâce à cette merveilleuse correspondance entre son heptachorde et la lyre des cieux, Orfée a pu apprivoiser les bêtes cruelles. Pour la première fois, Lefèvre de la Boderie cite le nom de Pythagore qu'il qualifie de "scavant" et de "divin." Celui-ci a su allier "la raison des nombres et des poids" grâce à son observation des marteaux, épisode rapporté par Macrobe, comme il l'indique, mais aussi par Boèce. Si celui-là a su formuler les rapports que l'on connaît - dit-il - entre les poids et les résonances, il n'a fait que les expérimenter car bien avant lui les Bardes avaient pénétré ce secret.

\section{La Musique et les quatre éléments}

Les Bardes ont même été plus loin dans leur découverte car non seulement ils ont révélé l'harmonie des cieux mais ils ont su également établir les fondements de la Musique humaine sur les quatre éléments:

Or non des Cieux sans plus, mais des quatre Eléments Nos Bardes empruntaient les quatre fondements de la Musique humaine. (p. 83 v) 
La Basse-contre représente la Terre, élément stable sur lequel s'appuient les autres éléments, la pleine Teneur ou seconde chorde renvoie à l'eau, le chant ou la Haute-contre fait penser à l'air et enfin au feu correspond la part qu'on nomme le Dessus. Or l'on connait les correspondances entre les quatre éléments, les humeurs, les saisons, les moments de la journée: chacun de ces quatre éléments jouissent de deux qualités physiques, ce qui permet de relier les éléments deux à deux et d'assurer un continuum si important dans le système de notre auteur:

\begin{tabular}{lc|} 
la terre & froid + sec \\
l'eau & froid + humide \\
l'air & chaud + humide \\
le feu & $\stackrel{\uparrow}{I}$ chaud + sec
\end{tabular}

Il est facile avec ses caractéristiques de relier les saisons aux quatre éléments:

$\begin{array}{ll}\text { hiver } & \text { froid }+ \text { sec } \\ \text { automne } & \text { froid }+ \text { humide } \\ \text { printemps } & \text { chaud + humide } \\ \text { été } & \text { chaud + sec }\end{array}$

Grâce aux analogies que l'on peut déduire entre les saisons, les différents moments de la journée et les différents âges, l'on peut établir de nouvelles correspondances. Ajoutons à cela les quatre humeurs: froide mélancolie, flegme humide, sang et bile:

Ils (nos bardes) pouvaient emprunter les pareilles raisons

Et de nos quatre humeurs, et les quatre saisons

Dont le tempérament accomplit et consomme

La consonance au Temps, et l'harmonie en l'homme. (p. 83 v)

Voilà donc le tetrachorde original des Bardes fondé sur les correspondances entre les cordes, les éléments, les humeurs et les saisons et que l'on schématise ainsi:

- chorde du Temps. feu Eté chaud et sec-bile chaude et sèche.

- Contre-haut - chant air printemps humide et chaud - sang aéré - moite

- la Teneur eau Automne humide-froid-flegme humide et froid

- Basse contre terre hyver froid-sec-froide mélancholie.

Au milieu de cet exposé "technique" nous sommes heureusement surpris de rencontrer quelques vers d'une bonne venue, nous en citerons quelques-uns 
qui révèlent un sens réel du rythme et du pittoresque que l'auteur sait mettre au service d'une pensée exigeante:

L'Este fait hérisser les plaines et les champs

D'espics murs barbelez, aux doux vents de panchants

Et flotants çà et là de leur perruque blonde

Comme on voit se suivir les branlements de l'onde. (p. 84 v)

Ces descriptions mettent en relief l'ordre, l'harmonie qui réside dans "ce grand corps" qu'est la terre et conduit le lecteur à découvrir parallèlement ce même ordre dans l'homme:

Le Tetrachorde humain contient aussi unie

Liant les quatre humeurs sa nombreuse harmonie. (p. 84 v)

Aux quatre temps de l'année correspondent les quatre parties du mois, les quatre moments de la journée, les quatre âges de la vie: l'enfance, la jeunesse, l'âge déclinant et la vieillesse. L'homme est un microcosme créé à l'image de l'univers. Guillaume Lefèvre de la Boderie a recours au Tikun Zohar comme il le signale en marge de son ouvrage. Il fait mention des vêtements qui recouvraient Adam. Avant la faute, ceux-ci étaient des vêtements de lumière כתנות אור (Kotnot 'or); après la faute, Dieu dans sa miséricorde a vêtu l'homme de vêtements de peau (כתנות עור) (Genèse III, 21). On remarquera ici que l'auteur fait quelques changements. Tout d'abord, par souci de cohérence(?), il introduit le nombre quatre ${ }^{10}$ et parle de vêtements d'une couleur dorée dans le premier cas, et de vêtements blancs dans l'autre. Autre changement plus important: le Zohar n'admet que trois degrés de l'âme humaine tandis que l'auteur de la Galliade paraît en admettre quatre. Il semble que l'âme naturelle dont il parle appartient, en fait, à la nature, soit à la matière, et correspond ainsi au corps: "Son Ame naturelle est sa Terre et son ombre." Les trois aspects de l'âme dont parle le Zohar sont nefesh (נטש) Iשמה (neshamah). Nefesh donne la vitalité et nous ne sommes guère étonnée de voir Lefèvre de la Boderie l'appeler la Vitale. Le degré supérieur est rua'h, Raison ou Esprit et enfin ce qui correspond à Neshamah est l'Entendement ou la Claire Pensée. Pour montrer la correspondance interne entre le macrocosme et le microcosme, l'auteur de la Galliade établit des parallèles entre les degrés de l'âme, les saisons et les humeurs:

La Vitale est son Eau, son soir, et son Automne, Son flegme, et second nerf, où l'autre voix s'entonne:

Son Esprit et son Air, son Printemps, son matin,

Son sang, sa tierce chorde au réson argentin.

Et l'Entendement haut, ou la claire Pensée 
Est son Feu, son my dour plein d'ardeur eslancé

Sa cholère enflamée, et son Esté plus chaut,

Et le nerf bien tendu qui sonne le plus haut. (p. 86, r)

La musique jouée sur ce luth résonne au Temple éternel. Sur le "throne" est assise la majesté de Dieu dont le nom, le tétragramme, "le nom quatrelettré," s'accorde "au quatrein de nostre Ame, ou à son Tetrachorde." Alors l'Entendement règne tout puissant sur les autres degrés de l'âme; la Raison obéit à l'Intellect, la "phantaisie" et le sens "élémentaire, obscur, meslé, mortel" se conforment à la Raison. Si la Raison ou Mèse se met à l'unisson avec la Nète ou Pensée, l'imagination et les sens s'accordent avec elles et l'on obtient l'harmonie. L'Esprit d'amour loue "le grand harpeur du Ciel,' l'Unité se réalise:

Et tirant la Raison moyenne à sa Neté

La lune à son Soleil, le deux à l'Unité. (p. 86, v)

Les quatre mondes sont harmonieusement unis:

Adong sont accordez entr'eux les quatre Mondes

L'humain, l'élémentaire et les Sphères profondes

Avec que l'archetype, et sonne l'Univers.

Une mesme harmonie en quatre luths divers. (p. 86 , v. et $87, \mathrm{r}$ )

L'on voit que la Boderie fait intervenir les quatre mondes, l'humain, l'élémentaire, les sphères profondes et l'archétype que l'on peut mettre en parallèle avec les quatre mondes de la mystique juive: monde de asiah איציצות עשיה (action), yetsirah (formation), beriah (création) et de atsiluth (émanation).

Au contraire, tout ce bel équilibre est détruit si " du pur Penser la Raison vers le Sens se vient à dispenser" tout est désaccordé et ainsi "est violé du Monde l'Hymenée." Guy Lefèvre de la Boderie en quelques beaux vers peint les effets de cette catastrophe dans la nature même:

Et l'Aigle bavolant sur ses Aiglons esclos

Pour les dresser au vol retourne en son repos,

Et les laisse seulets privez de toute grace

Ainsi qu'oiseaux bastards indignes de sa race,

Pour n'avoir peu long temps soustenir de l'oeil

Les rayons eslancez du supréme Soleil. (p. 87, r)

Reprenant un des thèmes de la mystique juive, l'auteur de la Galliade montre comment l'homme peut instaurer ou détruire l'équilibre si précieux non seulement en lui mais dans tout l'Univers: 
Si dong' l'homme est d'accord, le tout est accordant,

Et s'il discorde aussi, tout s'en va discordant. (p. 87, v)

\section{Les quatre systèmes}

La musique grecque n'était pas fondée sur ce seul tétrachorde originel. Le système musical grec était en fait composé de quatre tétrachordes: l'hypaton (le plus bas), le Meson (ou moyen), le diezeugménon et enfin l'hyperboleon. L'hypaton et le Meson ont une corde en commun ainsi que le Meson et le diezeugménon, nous obtenons ainsi une échelle de 14 sons auxquels il faut ajouter la proslambanomène au-dessous du tétrachorde hypaton, voilà composé le système parfait de quinze sons.

Dans une tentative de syncrétisme extrême, Guy Lefèvre de la Boderie va établir des parallèles avec trois autres systèmes. ${ }^{11}$ Au lecteur, qui a la patience de cheminer à travers ces pages et de suivre la démarche lourde de l'auteur, c'est tout l'Univers à la fois dans sa complexité mais aussi dans sa glorieuse unité qui apparaît. Celui-là connaîtra les secrets de l'Univers et il sera slors capable, non de maîtriser le monde - ce n'est point le but cherché par les Kabbalistes - mais d'accomplir la volonté de l'Etre suprême. Pendant le Moyen Age, les théologiens après avoir accepté l'existence des anges les subsituèrent aux muses. L'auteur de La Galliade a été frappé par cette rencontre qui, pour lui, n'est pas fortuite entre le système musical de 15 cordes et les 15 Psaumes de montée (psaumes 120 à 134 inclus) correspondants aux 15 marches qu'il y avait, selon la tradition juive, la Mishna, au Temple de Jérusalem menant de la cour des femmes à la cour des Israélites. C'est ainsi qu'il fonde son système sur le nombre 15 . Tous les théoriciens ne basent pas leur système sur le nombre 15 , d'autres le fondent sur le nombre 9 ( 3 x 3) ou 10; Robert Fludd, lui, édifiera le sien sur le nombre 22 (le monde a été créé avec les 22 lettres de l'alphabet hébraïque).

Il y a donc quatre systèmes parallèles chez Guy Lefèvre de la Boderie, comme nous pouvons le voir dans le tableau suivant: le système musical grec que, par chauvinisme, il appelle le système des Bardes, le système d'Orfée ou de l'univers, le système de David ou l'archétype et enfin le système humain ou microcosme. On peut lire ce tableau verticalement, de bas en haut ou de haut en bas, ou encore horizontalement, de gauche à droite ou de droite à gauche, selon que l'on parte de l'homme, ou de l'univers ou de Dieu. Dans son poème, notre auteur englobe tous les systèmes à la fois, montant la gamme de degré en degré. Par commodité, nous examinerons les systèmes séparément. (voir le table suivant.) 


\begin{tabular}{|c|c|c|c|c|c|}
\hline & $\begin{array}{l}\text { Système } \\
\text { des Bardes* }\end{array}$ & $\begin{array}{l}\text { Système } \\
\text { d'Orfée ou de } \\
\text { l'univers }\end{array}$ & $\begin{array}{l}\text { Système } \\
\text { de David ou de } \\
\text { l'archétype }\end{array}$ & $\begin{array}{l}\text { Système } \\
\text { humain ou du } \\
\text { microcosme }\end{array}$ & $\begin{array}{l}\text { Système } \\
\text { vocal }\end{array}$ \\
\hline 1) & $\begin{array}{l}\text { proslamva- } \\
\text { nomérée }\end{array}$ & $\begin{array}{l}\text { centre du } \\
\text { globe terrien }\end{array}$ & $\begin{array}{l}\text { chorde de } \\
\text { l'Estre }\end{array}$ & $\begin{array}{l}\text { sens du } \\
\text { touchement }\end{array}$ & $A$ (la) ré \\
\hline 2) & hypaté hypaton & terre & note du vivre & $\begin{array}{l}\text { sens du Goût } \\
\text { ni aqueux ni } \\
\text { terrestre }\end{array}$ & $\mathrm{B}$ (si) mi \\
\hline 3) & $\begin{array}{l}\text { parhypaté } \\
\text { hypaton }\end{array}$ & eau & note du sentir & sens du flairer & $C$ (ut) fa \\
\hline 4) & lichanos hypaton & air & $\begin{array}{l}\text { discours et } \\
\text { raisonnement }\end{array}$ & ouie & D (re) sol - l \\
\hline 5) & hypaté méson & feu & $\begin{array}{l}\text { chorde } \\
\text { d'entendre }\end{array}$ & vue & E (mi) la \\
\hline 6) & $\begin{array}{l}\text { parhypaté } \\
\text { méson }\end{array}$ & lune & $\begin{array}{l}\text { ordre des Saints } \\
\text { Anges } \\
\text { porte d'Adonaï } \\
\text { pour entrer } \\
\text { en Sion }\end{array}$ & $\begin{array}{l}\text { crainte de } \\
\text { Dieu }\end{array}$ & $F(f a) u t$ \\
\hline 7) & lichanos méson & mercure & $\begin{array}{l}\text { ordre des } \\
\text { Archanges } \\
\text { El-Shadaï semond } \\
\text { les Esprits à } \\
\text { monter en Sion le } \\
\text { double mont }\end{array}$ & piété & G (sol) té - ut \\
\hline 8) & Mésé & Vénus & $\begin{array}{l}\text { Principautés, } \\
\text { sous le nom de } \\
\text { Elohim Zevaoth }\end{array}$ & Science & $\begin{array}{l}\text { A (la) mi - re } \\
\text { Octave } \\
\text { double }\end{array}$ \\
\hline 9) & Paramésé & Phebus & $\begin{array}{l}\text { Ordre des } \\
\text { Puissances mues } \\
\text { par Iehovah } \\
\text { Zevaoth }\end{array}$ & $\begin{array}{l}\text { Force } \\
\text { courage }\end{array}$ & B (si) fa-b-mi \\
\hline 10) & $\begin{array}{l}\text { Trité de } \\
\text { Diezeugmenon } \\
\text { Tetrachorde } \\
\text { disjoint }\end{array}$ & $\begin{array}{l}\text { Mars mal } \\
\text { joint et } \\
\text { maléfique }\end{array}$ & $\begin{array}{l}\text { Vertus } \\
\text { Elohim }\end{array}$ & conseil & C (ut) sol-fa \\
\hline 1) & $\begin{array}{l}\text { Paraméte de } \\
\text { Diezeugmenon }\end{array}$ & Jupiter & $\begin{array}{l}\text { Dominations } \\
\text { Iehovah }\end{array}$ & Inteligence & D (re) la - sol \\
\hline 2) & $\begin{array}{l}\text { Neté de } \\
\text { Diezeugmenon }\end{array}$ & Saturne & $\begin{array}{l}\text { Ordre Saint des } \\
\text { thrones soutenu } \\
\text { par El, Dieu de } \\
\text { miséricorde }\end{array}$ & $\begin{array}{l}\text { haute } \\
\text { Sapience }\end{array}$ & $\mathrm{E}$ (mi) la \\
\hline 13) & $\begin{array}{l}\text { Trité de } \\
\text { Hyperboléon }\end{array}$ & $\begin{array}{l}\text { Ciel estellé } \\
\text { du monde } \\
\text { Firmament }\end{array}$ & $\begin{array}{l}\text { monde des Idées } \\
\text { l'ordre des } \\
\text { chérubins oracle } \\
\text { saint du Sacré } \\
\text { Iehovah - la } \\
\text { Trinité se fond } \\
\text { en unité }\end{array}$ & $\begin{array}{l}\text { Excellente } \\
\text { Foy }\end{array}$ & $F(f a) ~ u t$ \\
\hline 4) & $\begin{array}{l}\text { Parameté } \\
\text { d'Hyperboléon }\end{array}$ & $9^{\text {eme }}$ Sphère & $\begin{array}{l}\text { Seraphins } \\
\text { Iah }\end{array}$ & Espérance & G (sol) ré \\
\hline 15) & $\begin{array}{l}\text { Neté } \\
\text { d'hyperboléon }\end{array}$ & $\begin{array}{l}10^{\text {eme }} \text { ciel } \\
\text { ciel musical }\end{array}$ & $\begin{array}{l}\text { l'homme-Dieu- } \\
\text { Jésus Cercle infini } \\
\text { de la divinité. } \\
\text { Ehieh } \\
\text { Keter }\end{array}$ & Charité & A (la) mi-ré \\
\hline
\end{tabular}




\section{i) le système d'Orfée ou de l'univers}

Lefèvre de la Boderie doit se soumettre à deux impératifs: monter les 15 degrés de l'échelle et arriver au dixième ciel. Il lui faut donc partir avant la première planète reconnue alors, à savoir la lune. Dans certains systèmes la lune correspondait au proslambanomène, ici le premier degré de la gamme musicale correspond au centre du globe terrien, avant même les quatre éléments. Il y a une progression dans les éléments mêmes: le feu, au degré 5, en se volatilisant, nous permet de nous dégager de l'univers terrestre et de nous élever dans les cieux de planète en planète jusqu'au firmament, appelé aussi le ciel des étoiles fixes. Dans la cosmologie grecque, telle qu'on peut la trouver chez Avicenne, il y a un $9^{e}$ ciel ou empyrée. Par la suite les théologiens ajouteront un dixième ciel; Saint Thomas par exemple pense conformer ainsi la représentation du cosmos avec la Foi. Guy Lefèvre de la Boderie en fait le ciel musical. Il faut se rappeler que pour certains auteurs, ce n'est qu'à ce niveau que les anges jouaient de la musique et que dans la Commedia de Dante la musique ne se fait entendre qu'au paradis et ce n'est qu'au dixième ciel que toute une symphonie retentit.

\section{ii) le système de David ou archétype}

On remarquera que là aussi il y a une hiérarchie très rigoureuse. L'essence précède l'existence; les sens viennent avant la raison. La corde de l'Entendement nous permet de passer du monde proprement humain au monde supérieur, de même qu'au système d'Orfée on s'apprétait à se volatiliser avec le feu. A partir du sixième degré, Lefèvre de la Boderie se conforme très exactement à l'harmonie céleste que l'on a l'habitude d'attribuer à SaintDenis l'aéropagite: les anges, archanges, principautées, puissances, vertus, dominations, trônes, chérubins et enfin séraphins le plus proches de Dieu. Mais notre auteur a été initié à la Kabbale et à son système de sephiroth, c'est ainsi qu'il mentionne le mot keter כתר, au dixième grade. Il savait que les kabbalistes faisaient correspondre aux diverses sephiroth des noms divins différents, c'est ce qu'il fait comme nous pouvons le voir dans le tableau. On remarquera aussi que les trois derniers degrés de cette échelle mystique forment une catégorie supérieure dans laquelle la Trinité, nous dit Guy Lefèvre de la Boderie, se fond dans l'unité. Cette idée a sa source dans le De Verbo Mirifico de Reuchlin (celui-ci parle des trois noms ehei, hu et esh comme une manière de trinité représentant la suprême essence de Dieu). Au plus haut ciel, Dieu se révèle à l'homme sous le nom de Ehei אהיה; également règne Jésus, l'homme-Dieu qui a réussi l'expérience suprême puisque des enfers il a franchi tous les degrés de l'échelle mystique; c'est pourquoi tout l'univers, "le ciel dedans son rond, les enfers et la terre," lui rend hommage. 


\section{iii) le système humain ou microcosme}

Tout au bas de l'échelle se situent les cinq sens dans lesquels, malgré tout, l'on peut discerner une hiérarchie. On s'élève du sens du toucher, le plus grossier au sens de la vue, le plus délié, celui qui nous permet de passer à l'ordre supérieur, à savoir l'ordre moral: la crainte de Dieu. Dans le De Verbo mirifico, Reuchlin avait placé פ מחד pa'had (crainte) au premier échelon des séphiroth. Au niveau 9, nous parvenons au monde des vertus cardinales: force, conseil, intelligence sapience, et enfin, correspondant aux trois sephiroth supérieures nous nous hissons jusqu'à l'ordre des vertus théologales: Foy, Espérance, Charité.

\section{iv) le système vocal se conforme à l'usage en vigueur depuis Boèce}

Nous couvrons l'espace de deux octaves en partant du la correspondant au premier échelon du tétrachorde, le proslambamonème. De fait, ce système vocal ne paraît que dans la marge; il n'est là que pour guider les "praticiens" mais ne joue aucun rôle dans le système de concordances de Guy Lefèvre de la Boderie.

Ainsi grâce à ces correspondances mystérieuses, mais bien réelles aux yeux de Guy Lefèvre de la Boderie, l'homme à la possibilité de mettre en branle tous les systèmes à la fois, et s'élever de la matière la plus grossière à Jésus, l'homme-Dieu. La musique est le moyen le plus sûr parce que le plus technique; il suffit d'apprendre les lois de l'harmonie, des consonnances et dissonances. Grâce à ces lois l'on pourra faire naître la concorde universelle. A ce point Guy Lefèvre de la Boderie fait résonner la musique de toute la création dans un beau chant:

\section{Si vingt et quatre vieux}

Font résonner là haut des tons harmonieux Dans la Saincte Cité, avec lesquel s'assemble Le beau chant nuptial que font ouyr ensemble Les Astre du matin, qui d'hymnes tour à tour, Vont loulants le grand Dieu avant le poinct du jour:

Si dessus chaque Ciel s'assied une sereine, Qui sa sfere remplit de voix claire et sereine: Bref si de l'Univers (ainsi que veut Platon) Ceste Ame Archimusee, et qui meine le ton Dedans le ventre creux du Tout qu'elle dispose,

De nombres musicaux s'unit et se compose,

Se doit-on estonner si elle va formant

La voix harmonieuse en corps de l'Animant? (p. 91, v et 92, r)

La partie qui va suivre est une démonstration "pratique" des mystérieuses correspondances qu'il nous a révélées. Il s'agit de convaincre les musiciens 
empiristes des merveilleux effets de la musique afin qu'ils se mettent à l'oeuvre et produisent cette harmonie capable de faire descendre du ciel la paix si ardemment désirée.

\section{Les effets de la musique}

Lefèvre de la Boderie commence par montrer l'effet de l'harmonie, de la musique sur les animaux: oiseaux avec leurs jolis "fredons"; "le chardonneret":

\section{Et les gentes Linotes}

Accordent à l'envy leurs motets et leurs notes, Et que le Rossignol cadré dans les buissons

Harmonieusement gringote ses chansons. (p. 92, r)

Les abeilles aussi sont sensibles au "tintin de l'érain." Il conte la jolie anecdote où une cigale vint à suppléer le son d'une chanterelle qui, par accident, s'était rompue sur un luth. Les cerfs ailés se figent au son de la flûte et les éléphants de l'Inde sont eux aussi ravis par la musique. De la plus petite à la plus grosse, aucune créature ne saurait rester insensible à l'harmonie. Il ne manque pas de rappeler la légende d'Orfée qui par "ses fredons divins" attirait les bêtes et celle d'Arion qui menait les dauphins. Les pierres mêmes répondent au son des carmes et des chants (Amphion et la construction de Thèbe) et une source jaillit près de d'Eleusine si tôt que retentit le son de la flûte. Quittant les rivages méditerranéens, il nous entraîne en Finlande à la suite d'Olasgoth en l'histoire de Septentrion.

L'homme connait bien les effets de la musique: c'est le pasteur qui fait sauter ses troupeaux au son du chalumeau, le laboureur qui chantonne, le batelier, le charretier, l'enfant au berceau:

Mesme au berceau plorant le petit enfançon

Coy s'endort et s'appaise au bruit d'une chanson

Comme si l'Ame estant de Musique ravie

Rassembloit lors en un ses accords et sa vie. (p. 93, v)

Les sages, pénétrés des correspondances intimes entre les différents mondes, peuvent se servir de leur science pour guérir l'homme en proie à 'l'ire désaccordée." Lefèvre de la Boderie cite de nombreux exemples qu'il puise au livre des Dipnosophes et au traité de la musique de Plutarque. Des maladies plus organiques peuvent trouver leur cure dans la musique: Asclépiade guérissait les sourds au son de la trompette et du tambour, Hisménias "par douceur harmonique les fièvres guarissoit et goute sciatique," Thalès a su, grâce au pouvoir de la cythre, éloigner la peste et autres maux. Bien d'autres 
noms seraient à ajouter. Lefèvre de la Boderie fait appel également aux Hébreux pour démontrer les vertus cathartiques de la musique. David n'apaisait-il pas Saül en proie à la mélancolie? et au livre des Rois ne lit-on pas qu'Elisée fit venir un joueur de lyre (qu'il préfère appeler harpeur-celà a une consonnance plus hébraïque) afin de se rendre réceptif à l'esprit de Dieu?

Il n'est pas un théoricien qui ne croit en l'effet admirable de la musique, Platon (Lois, République), Aristote (Politique) en étaient persuadés; même un esprit moderne et rationaliste comme Mersenne ne met en doute ce pouvoir. ${ }^{12}$ Certains écrivains ont recours à des exemples plus modernes. Ainsi Pontus de Tyard relate l'anecdote dont fut témoin, dit-il, Monsieur de Vintimille séjournant à Milan (Solitaire Second). Bergier, dans La musique spéculative, affirme avoir user, lui-même - de la musique pour apaiser un jeune homme natif de Lorraine. Lefèvre de la Boderie cite le cas des "Azenegues noirs" qui furent "apprivoisés" par le doux son de la flûte. Si la musique calme les hommes, elle peut aussi les remplir d'humeur guerrière. Citons le cas des Spartiates luttant contre les Messéniens dont les coeurs furent rafermis grâce à Tirthée.

Lefèvre de la Boderie exhorte les musiciens du Roi - s'ils ont appris l'harmonie, c'est-à-dire s'ils savent "accorder la lyre dans les corps" - à tendre la corde de Raison et à ne laisser résonner la corde de l'ire que pour dénoncer les crimes, les forfaits “de qui va violant la Musique de Paix." Plus que jamais le Royaume de France a besoin de cette harmonie. Tout est dissonance, désaccord, les gentilhommes n'obéissent plus aux princes, les princes aux rois parce que les rois eux-mêmes n'obéissent au Roi suprême:

Ainsi l'ame est confuse au politique corps

Et le grand luth du Regne au perdu ses accords. (p. 96, v)

Que les musiciens chantent d'un ton sévère et grave, à la mode dorienne, la vraie Piété au Roi! Celui-ci, repentant comme le fut David, se rangera au service de Dieu et tout rentrera dans l'ordre. L'auteur de La Galliade ne ménage pas son souverain!

Les musiciens sauront ranimer le courage des capitaines en chantant d'un ton guerrier, à la mode phrygienne. Alors ceux-ci pourront repousser l'assaut de l'étranger ou du Français mutin. Ils ne feront que marcher sur les traces de leurs devanciers, les Bardes " bien appris." Voila bien la preuve que cet approfondissement des lois de l'harmonie universelle n'était pas pure spéculation, un divertissement de philosophe coupé de la réalité politique et sociale. Quant aux musiciens qui se contenteront de charmer les seigneurs et les dames, qu'ils prennent garde à choisir des accents chastes et purs:

N'entonnez en leurs coeurs, ny aux trous de leurs testes

Nuls tons qui ne soient purs, aetherez et celestes (p. 98, v)

point de "charnelle chanson qui Chasteté renverse." 
Non seulement le musicien doit connaitre les rapports harmoniques, mais il doit encore savoir choisir les modes sur lesquels composer sa musique. Lefèvre de la Boderie compte six modes: dorien, phrygien, écolien, ionnien ou iastien, lydien et myxolydien. On remarquera qu'il ne mentionne pas le lydien relâché $(\chi \alpha \lambda a \varrho \alpha \lambda v \delta ı \delta \tau \imath)$. Certains théoriciens de la Renaissance en comptent jusqu'à douze; c'est qu'ils doublent chaque mode d'une forme basse ou haute, ${ }^{13}$ mais tous admettent que les anciens ne connaissaient que sept modes qui correspondent aux sept espèces d'octaves. ${ }^{14}$ Chaque mode exerce une fonction particulière. Le mode dorien apporte la paix de l'âme tandis que le ton phrygien sera employé pour exhorter les guerriers (l'on voit qu'il y a confusion entre ton et mode). L'éolien amène au sommeil, l'ionnien porte l'âme à chercher les secrets divins, le lydien chasse les soucis et enfin le ton inventé par Sapho rend l'âme langoureuse.

Pour exalter l'âme, il faut lui donner la nourriture qui convient à sa nature, ${ }^{15}$ c'est-à-dire motets et cantique divins:

Rien de mol, rien de lasche, et rien au vice esclave,

Mais tout pur, tout constant, tout sonoreux et grave. (p. 100)

Dans un beau morceau lyrique, Lefèvre de la Boderie exalte la pudeur chez les damoiselles, comparant la chasteté à une fleur secrète. Toujours dans une belle envolée, il encourage les musiciens à célébrer la gloire de Dieu en reproduisant l'harmonie universelle:

\footnotetext{
Chantres, chantres, chantez du grand Dieu les louanges, Accordez vos veaux chants aux saincts hymnes des Anges, Gloire soit ès hauts lieux à l'unique Bonté

Paix en terre aux humains de bonne volonté

Faites-moy tournoyer par nombreuses parolles

Et les Esprits mouvants, et du Ciel les carolles:

Entonnez ès tuyaux des Orgues longs et ronds

Des Cieux organisez la Musique et les tons:

Faites sur le clavier d'une douce Espinete

Marcher d'orgue et de rang Planète après Planète

Sous le bal du grand Ciel ... (p. 100, v - 101, r)
}

ainsi imiteront-ils David qui sonnait de la harpe en la sainte cité. Que les musiciens décrochent leurs harpes des saules de Babylone pour entonner le chant nouveau; et pour les encourager, Guy Lefèvre de la Boderie donne lui-même l'exemple et laisse monter un chant de louanges.

\section{Doxologie}

Ce chant de louanges ou d'action de grâces court sur quelque neuf pages. En réalité il reprend dans une traduction proche du texte tout le Psaume 104 dans lequel David exalte Dieu dans sa création. L'auteur de la Galliade a su 
garder le mouvement de ce chant d'amour dont chaque mot est une prière et un tressaillement de joie. D'autres fragments de psaumes apparaissent aussi comme des versets des Psaumes 149 et 150. Dans cette doxologie finale, c'est tout un orchestre qui se trouve réuni pour louer l'Eternel dans son sanctuaire et dans le firmament.

Dans une ultime tentative pour fondre les diverses traditions, Lefèvre de la Boderie exhorte les musiciens du roi à émuler Iopas, le harpeur de Didon, qui rappelait à sa reine les enseignements d'Atlas. Gràce à Atlas, il peut renouer avec la légende de l'Hercule des Gaules qui avait retenu les leçons d'Atlas qui les tenait lui-même de Ianus (ou Noé). A son tour cet Hercule avait transmis les secrets à ses descendants, les Bardes gaulois.

\section{Conclusion}

Ainsi s'achève le quatrième chant de la Galliade, texte central de cette “épopée du savoir," car bien plus qu'un traité de musique, c'est toute une cosmologie que Guy Lefèvre de la Boderie nous propose. Mais il ne s'agit pas d'un froid exposé. L'auteur de la Galliade a intériorisé la doctrine platonicienne et la cabbale chrétienne. Il "sait"' les correspondances intimes entre le "petit monde" et le "grand monde"; il sait que l'homme, grâce à sa raison, peut reproduire l'harmonie de l'univers de même qu'il peut, par ignorance ou par malice, créer des accords discordants et amener de proche en proche le désquilibre dans toute la création. C'est pourquoi Guy Lefèvre de la Boderie a dévoilé tous les secrets de la musica mundana. Que les musiciens appliquent les lois de l'harmonie, grâce à leur musique et au processus de concaténation universelle, la paix étendra ses ailes tutélaires sur le monde, la volonté de Dieu sera faite et le Christ-Roi tégnera de nouveau sur la terre.

Il est difficile d'imaginer qu'à la même époque, Montaigne rédigeait les Essais, posant un des premiers jalons vers la pensée moderne et que quelques années plus tard Charron ferait inscrire sur sa maison de Condom "Je ne sçay." Guy Lefèvre de la Boderie, lui, ne met aucune idée reçue en question. Nous l'avons surpris, plusieurs fois, à reproduire des erreurs qui se répétaient depuis l'antiquité. Il ne lui vient point à l'esprit de soumettre certains faits à l'épreuve de l'expérience. Non, il est détenteur d'une "révélation," à charge pour lui de la transmettre de la façon la plus convaincante et si possible la plus poétique.

\section{Notes}

1 Cf. D.P. Walker, Musical Humanism in the 16th and early 17th Centuries, dans The Music Review, 2 (1941), 4.

2 Ms. Bib. Nat. fr. 1359 sans date, mais Bergier mourut en 1623.

3 Dans Advertissement aux lecteurs. 
4 Ayant à l'esprit ces différents rapports le lecteur peut comprendre aisément des vers que A.M. Schmidt considérait comme énigmatique, Cf. La Poésie scientifique en France au XVIe siècle, p. 255.

5 Soit $256 / 243$.

6 Livre I, chap. XXVII.

7 Notons que l'auteur attribue le même son aux planètes Vénus et Mercure. Cicéron expliquait cette identité de son à leur intensité ( $v i s$ ) commune; Macrobe par leur isodromie, toutes les deux tournant dans le même sens, d'est en ouest.

$8 \mathrm{Cf}$. Mersenne, L'Harmonie Universelle, livre premier des consonnances, p. 108.

9 Cela représente environ $22.300 \mathrm{~km}$.

10 Il est à noter que les prêtres, kohanim, portaient quatre sous-vêtements et quatre ornements vestimentaires (cf. Lévitique).

11 Nous retrouvons le nombre quatre dont nous avons vu l'importance aux yeux de l'auteur. On comprend pourquoi il traite de la musique, soit de l'univers, au cercle iv de son ouvrage.

12 "Quibus existimo me satis ostendisse quantam vim Graecorum musica tam Platonis, quam Aristotelis aetate habuerit, ut jam nemo sanae mentis ea de re dubitare debere vidatur" (Quaestiones in Genesim). Dans son livre de L'Harmonie Universelle, il affirme "qu'il est aisé de prouver que l'harmonie est utile à toutes les sciences" et il consacre la proposition X du livre III à expliquer l'utilité de l'harmonie dans la morale et la politique.

13 Ainsi Zarlino expose les 12 modes suivants: dorien, hypodorien, phrygien, hypophrygien, lydien, hypolydien, mixolydien, hypomixolydien, hyperdorien ou éolien, subhyperdorien, hyperphrygien appelé aussi Ionien ou iastien, et enfin subhyperphrygien.

14 Marin Mersenne établit ainsi les correspondances: mixolydien . . . F, lydien . . . E, phrygien ... D, dorien ... C, hypolydien ... B, hypophrygien . . . A et hypodorien . . . G.

15 Les anciens avaient déjà classé les modes. Platon prisait le mode dorien, hypodorien ou éolien ainsi que le phrygien pour leurs harmonies viriles et tranquilles. Le dorien parle à l'intelligence. Par ailleurs, il condamne le mode ionien parce qu'il amollit. Aristote pensait que le mode lydien convenait à l'éducation des jeunes gens. 